\title{
Prototype de thermobalance multitêtes avec cyclage thermique rapide
}

\author{
Sandrine Sureau, Daniel Monceau, Dominique Poquillon et Jean-claude Salabura ${ }^{a}$ \\ CIRIMAT, UMR CNRS 5085, Equipe MEMO, ENSIACET, 118 route de Narbonne, 31400 Toulouse, France
}

\begin{abstract}
Résumé - Lorsque la résistance à l'oxydation à haute température des matériaux est testée, l'essai d'oxydation cyclique est souvent utilisé comme référence car il intègre la cinétique d'oxydation isotherme, l'adhérence de l'oxyde, les contraintes mécaniques, le fluage de l'alliage métallique et de l'oxyde dans des conditions proches de celles de l'utilisation. Cet article présente un nouvel outil expérimental, qui permet la mesure simultanée et individuelle de la masse de plusieurs échantillons placés dans la même atmosphère contrôlée pendant le cyclage thermique rapide. Cette thermobalance multitêtes est décrite, en association avec la description de la méthodologie de mesure. Les performances de l'appareil sont données, ceci incluant les vitesses de chauffe et de refroidissement ainsi qu'un essai de mesure de masse continue sur un échantillon de Ni20Cr.
\end{abstract}

Mots clés : Oxydation cyclique / appareil de thermogravimétrie / four à lampe

\begin{abstract}
When testing the resistance to oxidation of high temperature materials, the cyclic oxidation test is used as a reference because it integrates isothermal oxidation kinetics, oxide scale adherence, mechanical stresses, metallic alloy and oxide mechanical behaviour in conditions close to the actual conditions of use. This paper presents a new experimental tool, which allows the simultaneous and individual measurement of the mass of several samples placed in the same controlled atmosphere during fast thermal cycles. This multi-sample thermobalance is described, in association with the description of the measurement methodology. Performances of the apparatus are given, including heating and cooling rates and continuous mass measurements test a Ni20Cr sample.
\end{abstract}

Key words: Cyclic oxidation / thermogravimetric apparatus / lamp furnace

\section{Introduction}

La résistance des alliages métalliques aux attaques environnementales combinées aux contraintes mécaniques se fonde sur leur capacité à former un oxyde protecteur, c'est-à-dire un oxyde avec une faible cinétique de croissance et une grande adhérence à l'alliage. La cinétique de croissance est dans un premier temps étudiée en utilisant les essais d'oxydation isotherme en laboratoire, et la nature et la microstructure de l'oxyde et du substrat sont déterminées par des observations post mortem des échantillons oxydés. L'adhérence de la couche d'oxyde est plus difficile à quantifier en termes de constantes physiques. Plusieurs méthodes ont été et sont encore développées, telle que le «inverted blister test »[1]. Mais généralement ce qui est observé sur les échantillons après refroidissement, ce n'est pas l'adhérence mais l'étendue de

\footnotetext{
a Auteur correspondant :

JeanClaude.Salabura@ensiacet.fr
}

l'écaillage. Celle-ci ne peut pas être directement déduit de l'adhérence de la couche d'oxyde car elle dépend également de l'intensité des contraintes (contraintes de croissance, contraintes dues à la différence entre les coefficients de dilatation du métal et de l'oxyde), de l'épaisseur et des propriétés mécaniques de l'oxyde et du substrat métallique. La quantification de l'écaillage peut être réalisée, par exemple, par l'analyse d'image de la surface de l'échantillon après refroidissement. Des essais spécifiques ont été également conçus pour évaluer la baisse de température critique pour qu'il y ait écaillage [2] et pour relier l'étendue de l'écaillage avec l'adhérence et les propriétés mécaniques. Enfin, les mesures de cinétique d'oxydation, d'adhérence de la couche d'oxyde, des contraintes de croissances à haute température, des propriétés mécaniques à haute température doivent être combinées avec une modélisation viscoplastique par éléments finis du système substrat/oxyde [3,4] afin de prédire si l'alliage va former un oxyde protecteur ou 
non. De plus, quand l'objectif est la prédiction de durée de vie à long terme, toutes ces mesures doivent être considérées comme fonctions du temps à cause de l'évolution de la microstructure et de la composition chimique de l'alliage sous la couche d'oxyde avec le temps, qui peuvent beaucoup affecter les propriétés mécaniques du revêtement, par exemple. Pour faire face à cette complexité, lorsque que la résistance à l'oxydation haute température du matériau est testée, les essais d'oxydation cyclique [5] sont souvent utilisés comme référence car ils intègrent la cinétique d'oxydation isotherme, l'adhérence de l'oxyde, les contraintes mécaniques, le fluage de l'alliage métallique et de l'oxyde induit par l'oxydation et leur évolution avec le temps, dans des conditions proches de celles d'utilisation. Ces résultats sont ensuite combinés aux résultats des essais de corrosion, érosion, fluage et fatigue à haute température afin de fournir un comportement général du matériau.

Pour relier les propriétés physiques (cinétique d'oxydation, contraintes de croissance, énergie d'interface, coefficient d'expansion thermique, propriétés mécaniques de l'alliage et de l'oxyde) aux essais d'oxydation cyclique, un travail scientifique de compréhension est nécessaire. Mais, des développements technologiques et de compréhension des essais d'oxydation cyclique sont encore à faire. L'oxydation cyclique est le plus souvent utilisée comme outil qualitatif ou « d'essai déterminant ». Deux voies ont été suivies pour améliorer cette quantification : 1) la modélisation de la cinétique [6-8], 2) le développement de nouveaux instruments ou de nouvelles méthodes de mesure. Ce papier s'intéresse au deuxième point en particulier avec l'utilisation de la thermogravimetrie en conditions d'oxydation cyclique.

\section{Thermogravimétrie en oxydation cyclique}

Pour quantifier la cinétique d'oxydation en condition cyclique, une méthode expérimentale a été développée afin de dissocier les dommages causés par l'oxydation isotherme et ceux dus à l'écaillage pendant le refroidissement (ou la remonté en température) [9]. Cette méthode est également consacrée à l'identification des paramètres nécessaires pour la modélisation de l'oxydation cyclique $[7,8]$. Elle est basée sur l'enregistrement en continu de la masse en fonction du temps pendant les paliers à haute température de l'essai d'oxydation cyclique réalisé dans une thermobalance. L'analyse des résultats proposée dans [9] fait l'utilisation de la série de points caractéristique $M_{\mathrm{n}}^{\text {bg }}$ et $M_{\mathrm{n}}^{\text {end }}$ (Fig. 1) pour calculer indépendamment la quantité d'oxyde formé et la quantité d'oxyde écaillé à chaque cycle.

A partir de l'enregistrement de la masse au début et à la fin des paliers à haute température et en faisant quelques hypothèses, plusieurs données sont accessibles : la variation de masse nette, le gain de masse total, la masse totale d'oxyde écaillé, la masse de métal consommé, l'épaisseur de la couche d'oxyde moyenne, la constante parabolique de croissance de la couche d'oxyde $\left(k_{p}\right)$ en

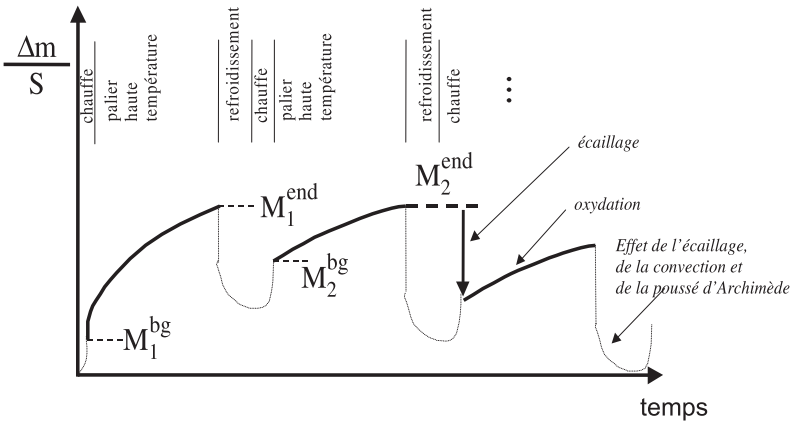

Fig. 1. Principe de la thermogravimétrie en oxydation cyclique [9].

fonction du nombre de cycles, et la fraction d'oxyde $\left(\mathrm{P}_{n}\right)$ qui s'écaille à chaque cycle.

La thermogravimétrie continue a plusieurs avantages par rapport aux essais avec mesure de masse discontinue pendant lesquels les échantillons doivent être placés dans des creusets afin de pouvoir mesurer indépendamment la variation de masse nette ainsi que la masse d'oxyde écaillée. En effet, les creusets sont une source de difficultés expérimentales $[10,11]$. De plus, avec la thermogravimétrie, les mesures sont obtenues automatiquement à chaque cycle. Néanmoins, le désavantage principal de cette technique est qu'elle est chère, car la majorité des études en oxydation cyclique nécessitent plusieurs échantillons pendant des temps d'essai très long (1000 à $20000 \mathrm{~h}$ ). Durant nos propres travaux effectués avec une thermobalance SETARAM ${ }^{\mathrm{TM}}$ classique $[9,12,13]$, nous avons fait l'expérience de ces difficultés expérimentales telles que les vitesses de chauffe et de refroidissement lentes, et la rupture d'un four après une centaine de cycles.

C'est pourquoi un appareil spécial a du être développé, permettant de tester plusieurs échantillons en même temps avec un gain économique lié à la réduction du nombre d'essai à réaliser pour une étude et à la réduction de manipulations.

\section{Développement d'une nouvelle thermobalance en oxydation cyclique}

Depuis plusieurs années, nous avons cherché à développer un nouvel appareil expérimental permettant de peser automatiquement plusieurs échantillons en même temps pendant qu'ils sont oxydés à haute température. Ceci devant être fait dans un appareil dont l'atmosphère peut être contrôlée (même pendant le refroidissement) et avec des vitesses de chauffe et de refroidissement rapides.

Le nouvel appareil, permet de tester cinq échantillons dans les mêmes conditions (de température, environnement, vitesse de chauffe, vitesse de refroidissement, durée des paliers chauds, durée des paliers froids). Chaque échantillon est suspendu au bout d'une ligne de pesée (Fig. 2-b).

Nous voulons que l'appareil atteigne des vitesses de chauffe et de refroidissement élevées $\left(>10{ }^{\circ} \mathrm{C} / \mathrm{s}\right)$ tout 


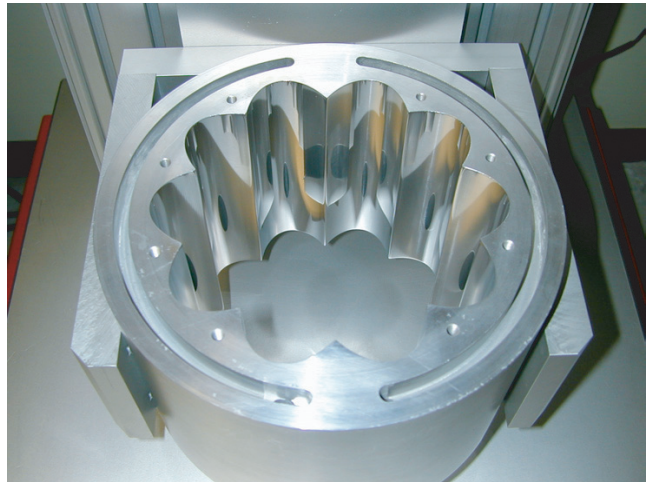

Fig. 2. Thermobalance multitêtes (a) cellules de pesées (b) échantillon suspendu à une ligne de pesée (c) pièce en alumine permettant de compartimenter l'enceinte du four.

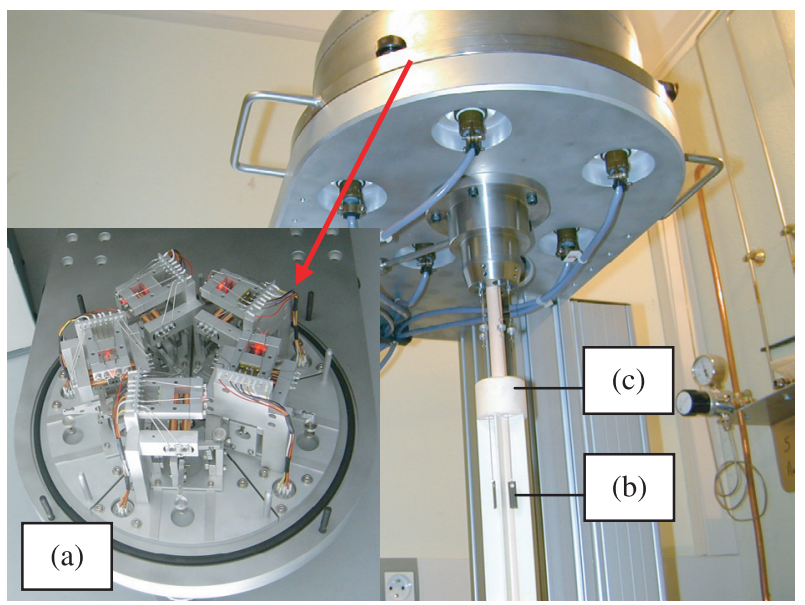

Fig. 3. Four à lampe 5 zones.

en permettant l'acquisition précise de la masse. Le four est donc un four à lampes appelé aussi four à image. Il est divisé en cinq zones (une par échantillon) qui sont indépendamment régulées en température. Pour chaque zone, nous avons deux demi-ellipses usinées dans la masse et polies jusqu'à obtenir une qualité miroir optique (Fig. 3). Ces deux ellipses ont un foyer « récepteur $»$ en commun où est placé l'échantillon. Des lampes de $750 \mathrm{~W}$ classiques sont situées sur les foyers « émetteurs ». L'enceinte du four est divisée en cinq chambres par une pièce en alumine (Fig. 2-c).

L'appareil est constitué de cinq cellules de pesée (Fig. 2-a) SETARAM d'une précision de 0,4 $\mu \mathrm{g}$ qui vont nous permettre de mesurer en continue les variations de masse des cinq échantillons oxydés. Il permet de travailler sous atmosphère contrôlée. Une entrée de gaz neutre a été placée au niveau de la cloche protégeant les cellules de pesée (en cas d'utilisation d'atmosphère corrosive) et une autre entrée de gaz oxydant placée sous le four (Fig. 4), la sortie du gaz s'effectuant au dessus du four (Fig. 4). Le système de mesure de masse étant très précis, il est très difficile de mesurer la température et la masse sur la même ligne. Donc la température est mesurée par des thermocouples $\mathrm{S}$ (Pt/Pt-Rh), un par échantillon, placés

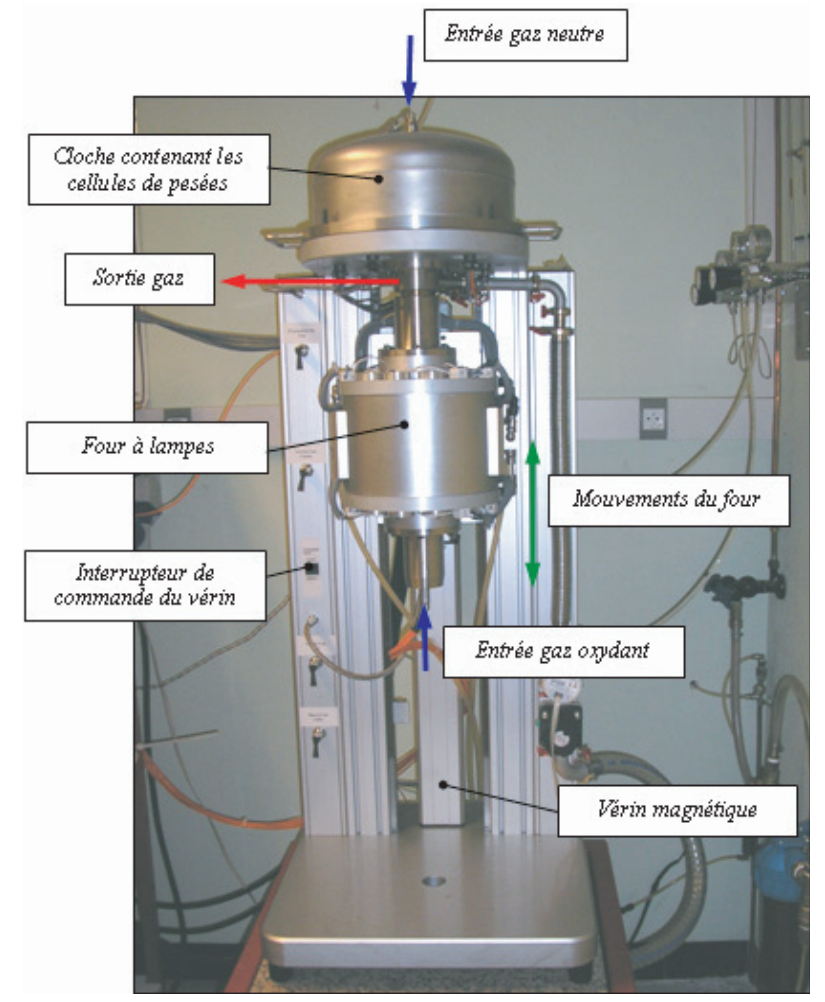

Fig. 4. Photographie du prototype de thermobalance multitêtes.

juste en dessous des échantillons (2 mm) à l'intérieur de témoins thermiques.

Afin de mettre en place et de retirer les échantillons de l'enceinte du four, celui-ci peut se déplacer verticalement. Ce mouvement est réalisé grâce à un vérin électrique que l'utilisateur peut facilement actionner à l'aide d'un interrupteur (Fig. 4). De plus la course du vérin est limitée par deux capteurs fins de course.

Enfin le pilotage de l'appareil se fait à partir d'un logiciel qui a été développé au laboratoire. Ce programme permet la programmation des Eurotherms ${ }^{\mathrm{TM}}$ qui régulent la température dans les cinq zones (un Eurotherm ${ }^{\mathrm{TM}}$ par zone) et l'acquisition des mesures de températures et de masses.

\section{Performances du prototype}

Des tests ont été réalisés afin de connaître les performances du prototype :

- La température maximum atteinte est de $1200{ }^{\circ} \mathrm{C}$;

- La vitesse de chauffe maximum pour un échantillon de $1,5 \mathrm{~mm}$ d'épaisseur est de $20^{\circ} \mathrm{C} / \mathrm{s}$ et celle de refroidissement de $10{ }^{\circ} \mathrm{C} / \mathrm{s}$ à $1000{ }^{\circ} \mathrm{C}$. Mais pour un échantillon de $100 \mu \mathrm{m}$ d'épaisseur la vitesse de chauffe peut atteindre $80{ }^{\circ} \mathrm{C} / \mathrm{s}$ et celle de refroidissement $25^{\circ} \mathrm{C} / \mathrm{s}$ pour la même température de régulation $\left(1000^{\circ} \mathrm{C}\right)$; 


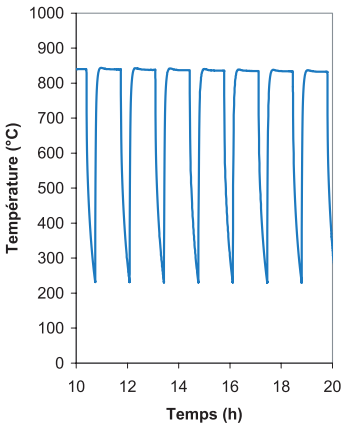

(a)

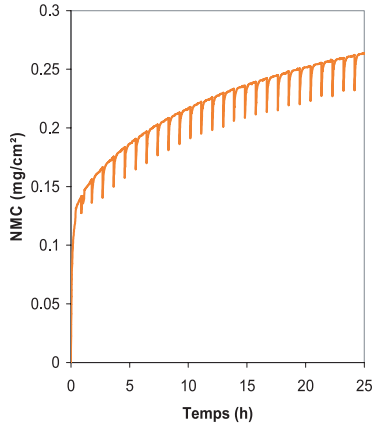

(b)
Fig. 5. Exemple d'essai d'oxydation cyclique sur un échantillon de Ni20Cr à $850{ }^{\circ} \mathrm{C}$ : (a) cycle thermique, (b) variation de masse.

- La température est la même à $\pm 1{ }^{\circ} \mathrm{C}$ sur tous les échantillons lorsque la température de régulation est atteinte;

- La précision de la mesure de masse est de quelques microgrammes.

Pour le cas beaucoup plus difficile des alumino-formeurs (alliages $\mathrm{NiAl}, \mathrm{FeAl}, \mathrm{FeCrAl}, \mathrm{NiCrAl}, \ldots$ ), les essais réalisés avec une thermobalance SETARAM ${ }^{\mathrm{TM}}$ TAG24S $[11,12]$ nous ont montré qu'une précision de $1 \mu \mathrm{g}$ était nécessaire pour traiter des essais avec un palier de $1 \mathrm{~h}$ à $1150{ }^{\circ} \mathrm{C}$ si nous voulons appliquer la méthode de thermogravimétrie cyclique proposée dans [9]. Néanmoins, même si la mesure de masse n'est pas assez précise pour utiliser cette méthode, l'évolution générale de la variation de masse nette en fonction du nombre de cycle est tout de même obtenue dans de très bonnes conditions. En effet l'atmosphère contrôlée et la mesure automatique à chaque cycle permettent d'obtenir des résultats du même types que pour des études réalisées précédemment [14-16] mais pour cinq échantillons et avec un four à lampe. La Figure 5 montre un exemple de cycle thermique et d'enregistrement de variation de masse pour l'oxydation d'un

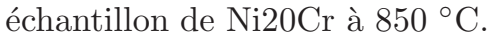

\section{Conclusion}

Afin de suivre en continu les variations de masses d'échantillons en oxydation cyclique, un prototype préindustriel a été conçu et construit. Il permet de chauffer des échantillons à une température de $1200{ }^{\circ} \mathrm{C}$ avec une vitesse élevée. La vitesse de refroidissement est du même ordre de grandeur que celle qu'il est possible d'obtenir en sortant les échantillons du four à haute température. Avec un four à lampes, il a été montré que la régulation thermique peut être excellente si les thermocouples de régulations sont placés dans des témoins thermiques. La combinaison d'un four à 10 lampes avec 5 échantillons pesés indépendamment par 5 balances dans la même atmosphère contrôlée apparaît déjà comme un outil avec un grand potentiel [17] pour quantifier l'essai d'oxydation cyclique (cinétique de croissance et écaillage à chaque cycle) et l'associer à la modélisation de la cinétique d'oxydation.

\section{Références}

[1] J. Mougin, M. Dupeux, A. Galerie, L. Antoni, Inverted Blister test to measure Adhesion Energy of Thermal Oxide Scales on Metals and Alloys, Materials Science and Technology, 18, 1217-1220 (2002)

[2] H.E. Evans, R.C. Lobb, Conditions For The Initiation Of Oxide-Scale Cracking and Spallation, Corrosion Science, 24, 209-222 (1984)

[3] H.E. Evans, A. Strawbridge, R.A. Carolan, C.B. Ponton, Creep effects on the spallation of an alumina layer from a NiCrAlY coating, Materials Science and Engineering, A225, 1-8 (1997)

[4] H. Echsler, E.A. Martinez, L. Singheiser, W.J. Quadakkers, Residual stresses in alumina scales grown on different types of $\mathrm{Fe}-\mathrm{Cr}-\mathrm{Al}$ alloys : effect of specimen geometry and cooling rate, Materials Science and Engineering, A384, 1-11 (2004)

[5] M. Schütze, W.J. Quaddakkers, Cyclic Oxidation of High Temperature Materials (EFC 27), The European Federation of Corrosion (1999)

[6] J.L. Smialek, Oxide morphology and spalling model for NiAl, Metallurgical and Materials Transactions, 9A, 309319 (1978)

[7] C.E. Lowell, C.A. Barrett, R.W. Palmer, J.V. Auping, H.B. Probst, COSP : a computer model of cyclic oxidation, Oxidation of Metals, 36, 81-112 (1991)

[8] D. Poquillon, D. Monceau, Application of a simple statistical spalling model for the analysis of high temperature cyclic oxidation kinetics data, Oxidation of Metals, 59, 409-431 (2003)

[9] D. Monceau, D. Poquillon, Continuous thermogravimetry in cyclic conditions, Oxidation of Metals, 61, 143-163 (2004)

[10] B.A. Pint, P.F. Tortorelli, I.G. Wright, Effect of cycle frequency on high-temperature oxidation behavior of alumina- and chromia- forming alloys, in : M. Schütze, W.J. Quadakkers (Eds.), Cyclic oxidation of high temperature materials, vol EFC 27, IOM Communications Ltd, London, 1999, pp. 111-132

[11] H.J. Grabke, D.B. Meadowcroft (Eds.), Guidelines for Methods of Testing and Research in High Temperature Corrosion (EFC 14), The European Federation of Corrosion, 1995, p

[12] D. Poquillon, D. Oquab, B. Viguier, F. Senocq, D. Monceau, High temperature oxidation kinetics of $\mathrm{NiAl}$ single crystal and oxide spallation as a function of crystallographic orientation, Materials Science and Engineering A, 381, 237-248 (2004)

[13] D. Poquillon, D. Oquab, D. Monceau, Cyclic oxidation kinetics modeling of NiAl single crystal, Materials Science Forum, 461-464, 737-745 (2004)

[14] J.C. Pivin, D. Delaunay, C. Roques-Carmes, A.M. Huntz, P. Lacombe, Oxidation mechanism of Fe-Ni-20-25Cr-5Al alloys. Influence of small amounts of yttrium on oxidation kinetics and oxide adherence, Corrosion Science, 20, 351$373(1980)$ 
[15] U. Krupp, S.Y. Chang, A. Schimke, H.J. Christ, Modelling internal corrosion processes as a consequence of oxide scale failure, in : M. Schütze, W.J. Quadakkers, J.R. Nicholls (Eds.), Lifetime modelling of high temperature corrosion processes, vol EFC 34, Maney Publishing, London, 2001, pp. 148-164

[16] P. Vangeli, B. Ivarsson, Investigation of a new methodology in high temperature oxidation. Application to commercial austenic steels., Materials Science Forum, 369-372, 785-792 (2001)

[17] Dispositif et procédé de test, par thermogravimétrie, du comportement d'un matériau solide in : vol $\mathrm{N}^{\circ}$ enregistrement francais : FR0300742, $\mathrm{N}^{\circ}$ demande internationale : PCT/FR2004/000090, $\mathrm{N}^{\circ}$ publication internationale : WO2004/068102, $\mathrm{N}^{\circ}$ demande brevet canadien CA2514070, N $^{\circ}$ demande brevet Europe EP1585976. 